\title{
Image Decomposition by Radial Basis Functions
}

\author{
Jens D. Andersen \\ Department of Computer Science, \\ Universitetsparken 1 , \\ DK-2100 Copenhagen $\varnothing$
}

\begin{abstract}
In order to reconcile linear and morphological scale space an image decomposition based on radial basis functions (RBF's) may be used. Radial-basis functions can be used to synthesize approximations of multidimensional functions, i.e. solving the problem of hypersurface reconstruction thus approximating the image intensities. They can be used in several ways in RBF networks which are linear neural networks. They provide a link between linear and morphological scale spaces. A morphological scale-space is a scale dependent decomposition of images from coarse to fine scale based on morphological operations on images. This is in contrast to the linear scale space, which is based on gaussian smoothing of image features. Both types of scale space are being advocated for image segmentation.
\end{abstract}

Keywords: Scale space, radial basis functions, neural networks.

\section{Introduction}

In order to reconcile linear and morphological scale space an image decomposition based on radial basis functions (RBF's) may be used. Linear scale space is obtained by convolving with a Gaussian kernel. After convolution the image is blurred and areas of interest, like edges, become smeared. Moreover, the localisation of areas of interest is changed in the image. Therefore, when linear scale space is used as a segmentation aid, e.g. by watershed partitioning of uniform areas, it is required to link region boundaries across scales exploiting the deep structure of the scale space [9]. Considering morphological (or non-linear) scale space, there exists several approaches to this. One proposal is morphological scale space with levelings [6], which has been shown not to displace region boundaries by transformation from one scale to the next (called leveling), while preserving other useful features of scale space simplification.

In order to combine linear and non-linear scale space it is advantageous to have a scale-based decomposition of images. Radial basis functions provide a basis for such a fine-to-coarse decomposition.

\section{Radial Basis Functions and Multi-layered Networks}

A method suggested by Poggio and Girosi [10] for image representation by multileayered networks (MLNs) is paving the $2 \mathrm{D}$ input sensor array space by RBFs 

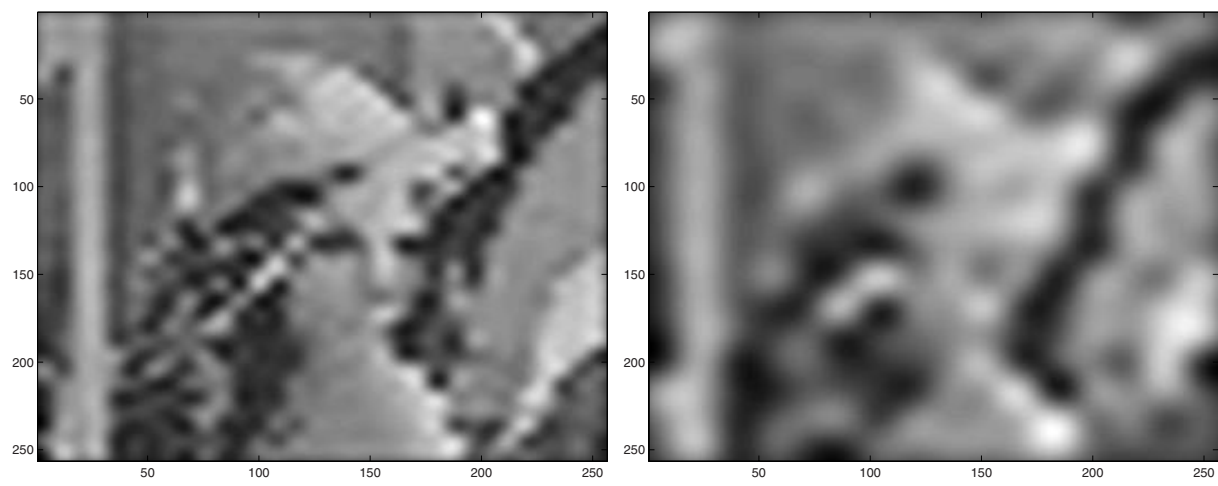

Fig. 1. Reconstruction of the 'Lena' image from RBF-decompositions. Left: scale $s=8$ (1024 weights), right: scale $s=16$ (256 weights) (Original image $256 \times 256$ pixels). Regularisation parameter $\lambda=0$.

and to use these in decompositions of arbitrary 2D stimuli into elementary 'blobs' of varying size. One interesting property of RBFs is their scalability. General RBFs are scaled by a scale parameter $s$. By the choice of $s$ it is possible to determine the influence of difference between the actual and the target pattern on the parameters propagated further on in the network. Thus it is possible to perform multiresolution analysis of images in the first layer of a neural net. The strategy of combining RBF coefficients at various levels of resolution may have a biological correlate in that there is evidence that shape perception in mammalians is performed by combination of output from columnar organized tuned receptive fields of varying size [2]. The problem of scaled decomposition of images may be viewed as an interpolation problem. A smooth approximation to a set of data is possible by using the regularization approach [10]. A regularization formulation leading to RBFs is the following: The goal is to guess or estimate a function which best describes the image intensity distribution (the output) from pixel coordinates (the input) with no knowledge of the form of the function. This problem has different names in different disciplines (nonparametric regression, function approximation, system identication, inductive learning etc.)(see [7]). Figure 1 shows a reconstruction of an image from RBF-decompositions at two different scales.

In the neural network formulation it is the supervised learning problem: The function is learned from a training set, consisting of paired values of the independent (input) variable and the dependent (output) variable. In the functional relation $y=f(\mathbf{x}) \mathbf{x}$ is a vector (pixel coordinates) and the dependent variable (the corresponding image intensity value) $y \in I$ is a scalar. The training set consists of $p$ pairs $\left(\mathbf{x}_{i}, \hat{y}_{i}\right), i=1, \ldots, p$, where the input points are chosen at random or according to some predetermined pattern. Here we use a quadratic grid with grid points $\xi_{j}$ spaced according to the scale $s$. 
A linear model for the function $y(\mathbf{x})$ has the form

$$
f(\mathbf{x})=\sum_{j=1}^{m} w_{j} h_{j}(\mathbf{x}) .
$$

Thus the model is a linear combination of a set of $m$ fixed basis functions using weights $w_{j}$. As basis functions among several possible RBF's Gaussian RBF's have been chosen:

$$
h_{j}\left(\mathbf{x}_{i}\right), j=1, \ldots, m, i=1, \ldots p, \text { with } h_{j}\left(\mathbf{x}_{i}\right)=A \cdot e^{-\frac{\left(\mathbf{x}_{i}-\xi_{j}\right)^{2}}{s^{2}}}
$$

With supervised learning and linear models a least squares principle leads to the following optimization problem: determine, using a training set $\left(\mathbf{x}_{i}, \hat{y}_{i}\right), i=$ $1, \ldots, p$

$$
f(\mathbf{x})=\sum_{j=1}^{m} w_{j} h_{j}(\mathbf{x}) \text { such that } S[f]=\sum_{i=1}^{p}\left(\hat{y}_{i}-f\left(\mathbf{x}_{\mathbf{i}}\right)\right)^{2}
$$

(the sum of squares) is minimized (by finding the optimum weights).

It is also possible to formulate this as a regularisation problem by adding a weight penalty term to the sum of squares:

Determine the function $f$ that minimizes the functional (the cost function)

$$
C[f]=\sum_{i=1}^{p}\left(\hat{y}_{i}-f\left(\mathbf{x}_{\mathbf{i}}\right)\right)^{2}+\sum_{j=1}^{m} \lambda_{j} w_{j}^{2},
$$

where $\left\{\lambda_{j}\right\}_{j=1}^{m}$ are regularisation parameters.

Minimisation of the cost function leads to a set of $m$ simultaneous linear equations in the $m$ unknown weights $w_{j}$. This may be written as a matrix equation (see [7] ):

$$
\mathbf{A} \hat{\mathbf{w}}=\mathbf{H}^{T} \hat{\mathbf{y}}
$$

$\mathbf{H}$ is called the design matrix, and $\mathbf{A}^{-1}$, the variance matrix, is

$$
\mathbf{A}^{-1}=\left(\mathbf{H}^{T} \mathbf{H}+\boldsymbol{\Lambda}\right)^{-1},
$$

where the $\boldsymbol{\Lambda}$-matrix is diagonal, and the elements along the diagonal are the regularisation parameters.

Given the vector of training set outputs $\hat{\mathbf{y}}=\left[\hat{y}_{1}, \hat{y}_{2}, \ldots, \hat{y}_{p}\right]^{T}$ the weights are found by

$$
\hat{\mathbf{w}}=\mathbf{A}^{-1} \mathbf{H}^{T} \hat{\mathbf{y}},
$$

The reconstructed approximating functions (the decomposition at the given scale $s$ ) is thus given by:

$$
f^{s}(\mathbf{x})=\sum_{j=1}^{m} w_{j} h_{j}^{s}(\mathbf{x})
$$


Multiscale decomposition is obtained by initial decomposition at a coarse scale, followed by subtraction of the reconstructed function at this scale from the original image $I$. Subsequently the residual $R(s)$

$$
R(s)=I-f^{s}(\mathbf{x})
$$

is decomposed at the next finer scale, continuing in this way until the desired approximation has been obtained.
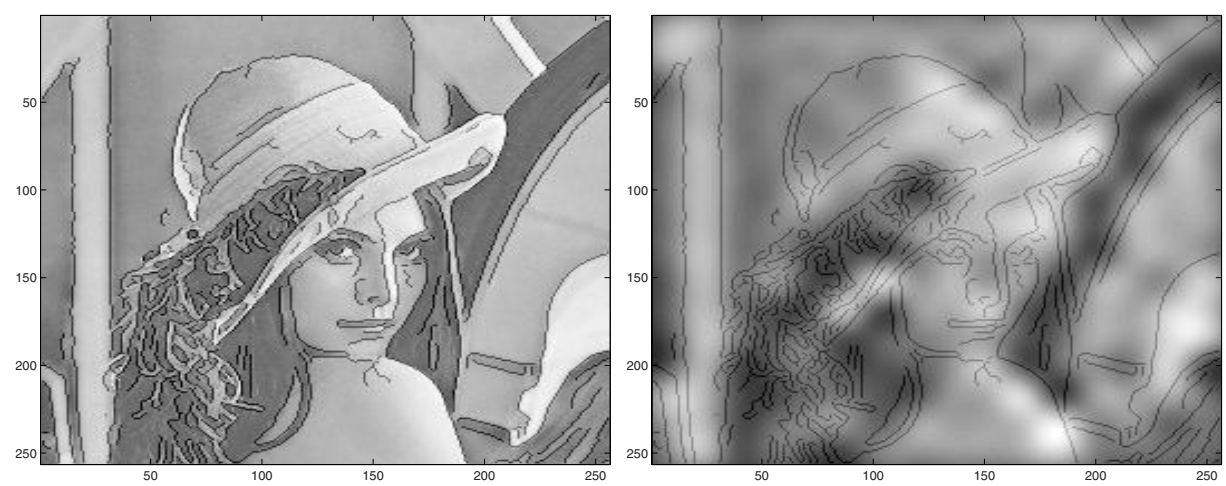

Fig. 2. Left: The 'Lena' image after leveling superimposed with edges derived from the leveling by the Canny edge-detector. Right: Leveling boundaries and regions marked by RBF's at scale $s=16$.

\section{Relation to linear scale space}

With this multiscale decomposition the image intensity approximation $I^{*}$ is given as a linear combination of approximating functions $\left\{f^{s_{i}}\right\}$ :

$$
I^{*}=\sum_{i=1}^{k} f^{s_{i}},
$$

where each $f^{s_{i}}$ in turn is a linear combination of RBF's at different scales. The effect of Gaussian blurring (i.e. applying a linear scale space transformation) corresponds to a similar transformation of the individual RBF's, while the linear combination remains invariant. Thus the decomposition is preserved in scale space, although the constituent RBF's are modified in a predictable way. This is because the result of convolving a Gaussian kernel with a Gaussian kernel is another Gaussian kernel

$$
g(\cdot ; t) * g\left(\cdot ; s_{i}\right)=g\left(\cdot ; t+s_{i}\right),
$$


i.e. a representation at a coarse scale $I^{*}\left(\cdot ; t_{2}\right)$ can be computed from a representation at a finer scale $I^{*}\left(\cdot ; t_{1}\right)$ by convolution with a Gaussian kernel with parameter value $t_{2}-t_{1}>0$

$$
I^{*}\left(\cdot ; t_{2}\right)=g\left(\cdot ; t_{2}-t_{1}\right) * I\left(\cdot ; t_{1}\right),
$$

This cascade smoothing property is due to the semi-grup property of the family of Gaussian kernels $[5,11]$

\section{Relation to morphological scale space}

Non-linear scale-space representations, frequently in the form of morphological scale-space representations, have been advocated. The argument for using morphological (non-linear) scale-space rather than Gaussian scale-space has been preservation of contours (essential for the subsequent segmentation process), and no creation of spurious extrema, as may occur in Gaussian scale space. In fact, when performing segmentation based on Gaussian scale space it may be required to track down contours to scale zero (the original image) as shown by Olsen $[8,9]$ (multi-scale linking of events).

One promising non-linear scale-space representation proposed by Meyer and Maragos is multiscale representation of images through levelings [6]. The basic idea is simple: by a stepwise 'flattening' of the image irrelevant information (noise) is discarded while important structural features are retained. More precisely: an image $\bar{I}$ is a leveling of an image $I$ if and only if for all neighbour pixels indexed by $\left(\mathbf{x}_{1}, \mathbf{x}_{2}\right): I\left(\mathbf{x}_{1}\right) \geq \bar{I}\left(\mathbf{x}_{2}\right)$ and $\bar{I}\left(\mathbf{x}_{1}\right) \geq I\left(\mathbf{x}_{2}\right)$. The relation " $(I)$ is a levelling of $I$ " is written $\bar{I} \prec I$. However, image segmentation based on morphological multiscale representation with levelings requires markers (see [6]). Various structuring elements have been tried, but the real problem is how to perform uncommitted marking, i.e. without any prior assumptions about region shapes. Here RBF-based decomposition may be used for the initial marking before the morphology-based image segmentation. An example of this use of RBF's is shown in figure 2 .

\section{Discussion}

In recent years numerous efforts have been made to try to reconcile the two fundamentally different multiscale representation of images: the linear and the non-linear (morphological) scale space. In spite of theoretical formulations uniting the two different approaches $[1,3]$ very little has been put to practical use yet. Different research groups advocate one or the other type of scale space as the tool for image segmentation.

An image decomposition with structural properties, which are predictably modified by different scale-space representations, linear and non-linear, is desirable. One such decomposition is offered by radial basis functions. The decomposition is preserved in linear scale space and may serve as region marking tool in non-linear (levelling) scale space. Thus the present communication is an attempt at a practically oriented 'intersection' of the two different worlds. 


\section{References}

1. L. Alvarez, F. Guichard, P.-L. Lions and J.-M. Morel: Axioms and fundamental equations of image processing. Arch. Rational Mech. Anal. vol. 123, pp. 199-257, 1993.

2. I. Fujita, K. Tanaka, M. Ito and K. Cheng, Columns for visual features of objects in monkey inferotemporal cortex, Nature, vol. 360, pp. 343-346, november 1992.

3. H.J.A.M. Heijmans and R.v.d. Boomgaard: Algebraic Framework for linear and morphological scale-spaces. Journal of Visual Communication and Image Representation, vol. 13(1/2), pp. 269-301, March 2002.

4. J. Froment: A compact and multiscale image model based on level sets. In: ScaleSpace '99 (M. Nielsen et al, eds.), Berlin: Springer-Verlag: LNCS 1682, pp. 152-163, 1999.

5. T. Lindeberg: Scale-space theory in computer vision. Doordrect, Kluwer Academic Publishers 1994.

6. F. Meyer and P. Maragos: Morphological scale-space representation with levelings. In: Scale-Space '99 (M. Nielsen et al, eds.), Berlin: Springer-Verlag: LNCS 1682, pp. 187-198, 1999.

7. Mark J.L. Orr: Introduction to Radial Basis Function Networks. Technical Report, Centre for Cognitive Science, University of Edinburgh 1996. www.anc.ed.ac.uk/ mjo/papers/intro.ps

8. O.F. Olsen: Generic image structure. Ph.D.-thesis. Department of Computer Science, Faculty of Science, University of Copenhagen 2000.

9. O.F. Olsen and M.Nielsen: Generic events for the gradient squared with application to multi-scale segmentation. In: B. ter Haar Romeny et al: Scale-Space Theory in Computer Vision 1997, pp. 101-112, Berlin: Springer-Verlag: LNCS 1252, 1997.

10. T. Poggio and F. Girosi, Networks for Approximation and Learning, Proc. IEEE, Vol. 78, No. 9, pp. 1481-1497, September 1990.

11. J. Sporring et al.:: Gaussian scale-space theory. Doordrect, Kluwer Academic Publishers 1997. 